\title{
ON THE CHARACTERISTIC EQUATIONS OF CERTAIN MATRICES
}

\section{ALFRED BRAUER}

In a paper to be published soon in the Annals of Mathematical Statistics, R. v. Mises obtains the following theorem on matrices from results in the theory of probability.

TheOREM. Let $A=\left(a_{\kappa \lambda}\right), B=\left(b_{\kappa \lambda}\right)$, and $C=\left(c_{\kappa \lambda}\right)$ be square matrices of order $n$. If the elements of $A$ and $C$ satisfy the conditions

$$
\begin{array}{rlrl}
r_{k} & =\sum_{\nu=1}^{n} a_{k \nu}=0 & (\kappa=1,2, \cdots, n), \\
s_{\lambda}=\sum_{\nu=1}^{n} a_{\nu \lambda}=0 & (\lambda=1,2, \cdots, n), \\
c_{k \lambda}=c_{k}+c_{\lambda} & (\kappa, \lambda=1,2, \cdots, n)
\end{array}
$$

where $c_{1}, c_{2}, \cdots, c_{n}$ are arbitrary numbers, then the matrices $A B$ and $A(B+C)$ have the same characteristic equation.

In the following a purely algebraic proof of this theorem will be given.

Proof. We set

$$
\sum_{\nu=1}^{n} a_{\kappa \nu} c_{\nu}=q_{\kappa} \quad(\kappa=1,2, \cdots, n) .
$$

Then we have by (1) and (3)

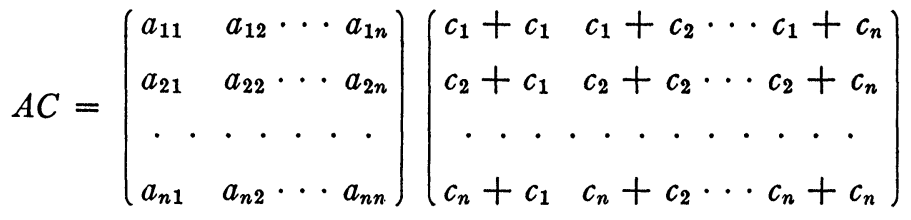

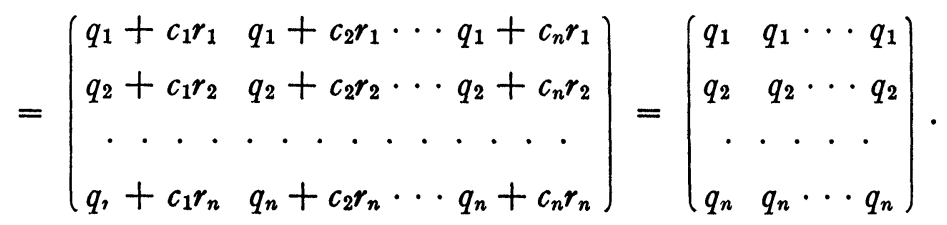

Let $P$ be the triangular matrix

Received by the editors December 2, 1946. 


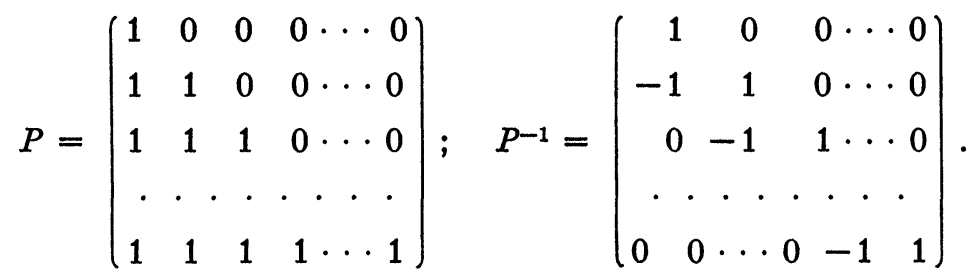

We have by (4)

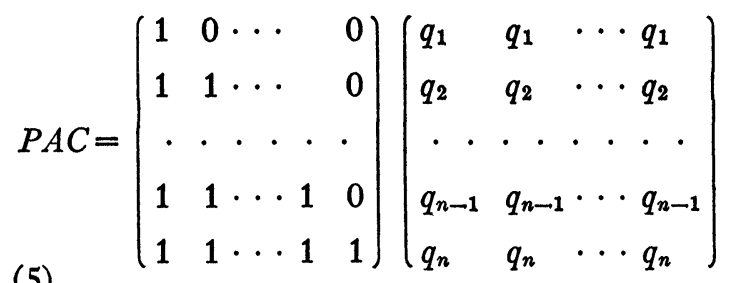

(5)

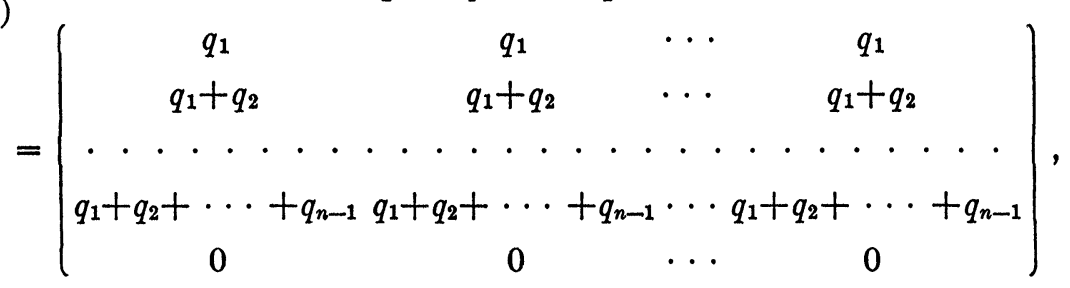

since by (2)

$$
\sum_{\nu=1}^{n} q_{\nu}=\sum_{\nu=1}^{n} \sum_{\lambda=1}^{n} a_{\nu \lambda} c_{\lambda}=\sum_{\lambda=1}^{n} c_{\lambda} \sum_{\nu=1}^{n} a_{\nu \lambda}=\sum_{\lambda=1}^{n} c_{\lambda} s_{\lambda}=0 .
$$

Hence

(6)

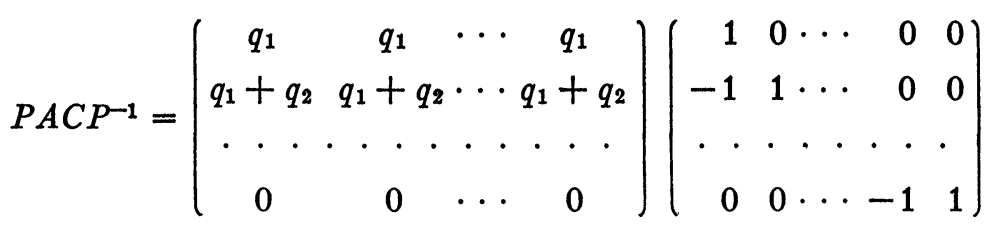

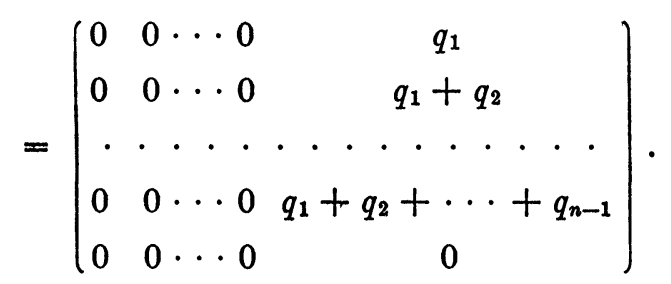

On the other hand, it follows from (2), similarly as in (5), that $P A$, and therefore also $P A B$ and $P A B P^{-1}$, are matrices in which all the 
elements of the last row are equal to 0 . Hence $P A B P^{-1}$ has the form

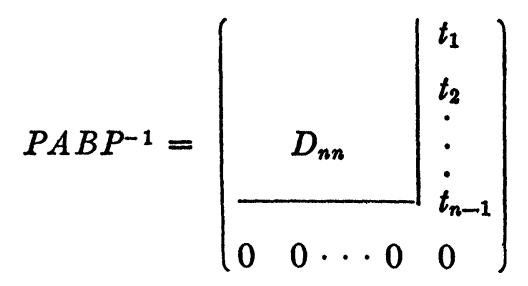

where $D_{n n}$ is a square matrix of order $n-1$ and $t_{1}, t_{2}, \cdots, t_{n-1}$ are certain elements. It follows from (7) and (6) that

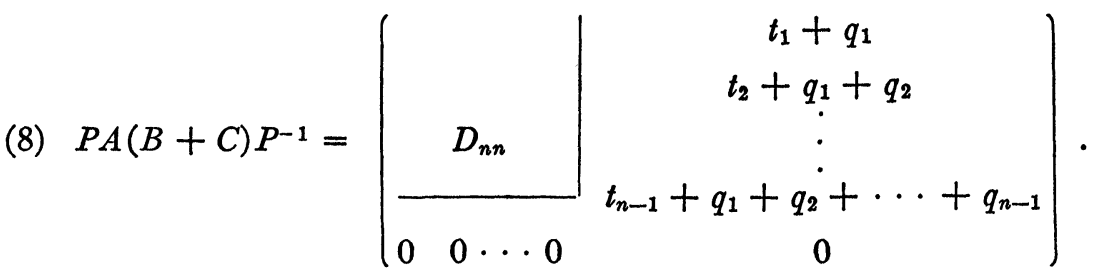

If we denote the characteristic polynomial of the matrix $D_{n n}$ by $f(x)$, then it follows from (7) and (8) that $P A B P^{-1}$ and $P A(B+C) P^{-1}$ both have the characteristic equation

$$
x f(x)=0 .
$$

Since similar matrices have the same characteristic equation, (9) is also the characteristic equation of $A B$ and $A(B+C)$.

University OF North Carolina 